\title{
ANDERSON AND GORENSTEIN DUALITY
}

\author{
J.P.C.GREENLEES AND V.STOJANOSKA
}

\begin{abstract}
The paper relates the Gorenstein duality statements of DGI06, DGI11] to the Anderson duality statements of [Sto11, Sto12], and explains how to use local cohomology and invariant theory to understand the numerology of shifts in simple cases.
\end{abstract}

\section{Contents}

1. Introduction

2. Anderson duals

1

3. The Gorenstein condition

4. Gorenstein duality and Anderson self-duality

5. Examples with polynomial or hypersurface coefficient rings

6. Invariant theory and descent

References

\section{InTRODUCTION}

1.A. Motivation. This paper emerged from a desire to understand the relationship between the duality statements that the two authors had been working on. More precisely, we wished to relate the Gorenstein duality statements of [DGI06, DGI11] to the Anderson duality statements of [Sto11, Sto12]. It was clear they were closely related, but here we make the relationship precise.

One of us had been considering connective ring spectra $\mathbf{r}$ (such as $k u$ or $\operatorname{tm} f_{1}(n)$ ) and proving when they have Gorenstein duality, and one of us had been considering non-connective spectra $\mathbf{R}$ (such as $K U$ or $T m f_{1}(n)$ ) and proving when they are Anderson self-dual. In many cases of interest, it is easy to recover $\mathbf{r}$ as the connective cover of $\mathbf{R}$, but also in favourable cases $\mathbf{R}$ can be recovered from $\mathbf{r}$ by a well known

We are grateful to MSRI and MPI for giving us the opportunity to start these discussions, to the referee for careful reading and detailed comments, to J.Rognes for an email conversation suggesting the connection described in Subsection 3.F and to C.Rezk about further discussion regarding that connection. The second author thanks the NSF for support through grant DMS-1606479. 
localization process, and under these processes the dualities correspond (Proposition 4.1 and Lemma 4.3).

In many cases $\mathbf{r}$ can immediately be seen to have Gorenstein duality since the coefficient ring $\mathbf{r}_{*}$ has it. Similar reasoning on the level of coefficients then gives that $\mathbf{R}$ is Anderson self-dual.

Furthermore in many cases there is a Galois-like action of a finite group $G$ on $\mathbf{r}$ and on $\mathbf{R}$, which is compatible with the process of moving from $\mathbf{r}$ to $\mathbf{R}$ and back again. (In the above cases $G$ is $C_{2}$ or $(\mathbb{Z} / n)^{\times}$). In favourable cases the fixed point ring spectra $\mathbf{r}^{G}$ are of interest ( $k o$ or $t m f_{0}(n)$ ). Furthermore, the action of $G$ on $\mathbf{R}$ is Galois with fixed point spectrum equal to the homotopy fixed point spectrum, and $\mathbf{R}^{G} \simeq \mathbf{R}^{h G}$ is also of interest ( $K O$ or $T m f_{0}(n)$ ). It may happen that the Gorenstein duality of $\mathbf{r}$ descends to that of $\mathbf{r}^{G}$, or that the Anderson self-dualiy of $\mathbf{R}$ descends to that of $\mathbf{R}^{G}$, but even when this happens the shift will change.

The simplest case is when the group order is invertible, so that the coefficients of the homotopy fixed points are the invariants: $\mathbf{R}_{*}^{h G}=\left(\mathbf{R}_{*}\right)^{G}$, and we point out here that in this case character theory often predicts the change in shift.

In general these examples come in fours: $\mathbf{r}, \mathbf{R}, \mathbf{r}^{G}$ and $\mathbf{R}^{G}$. One may hope to prove duality (in cases where it holds) by the following route: (1) we have duality for $\mathbf{r}_{*}$ and hence for $\mathbf{r}$ (2) we infer duality for $\mathbf{R}$ (3) we obtain duality for $\mathbf{R}^{G}=\mathbf{R}^{h G}$ by descent and (4) we infer duality for $\mathbf{r}^{G}$. The contents of this paper deal with the step from (1) to (2) and from (3) to (4). The step from (2) to (3) is more subtle and more interesting, and we hope to return to it elsewhere. The interested reader can find specific examples of this step in [HS14, HM17, Sto12]; related is the step (1) to (4), worked out in specific examples in GM16].

Beyond $K$-theory, our examples come from derived algebraic geometry. In this setting, it is the spectra $\mathbf{R}$, rather than the connective $\mathbf{r}$, which are primordial. In the presence of a gap in the homotopy groups of $\mathbf{R}$, one gets $\mathbf{r}$ as the connective cover. Unfortunately, there is no known procedure for obtaining $\mathbf{r}$ from $\mathbf{R}$ in wide generality, other than the ad hoc strategies that Hill-Lawson [HL10] and Lawson Law15 have employed. One could dream of an approach to connective covers which integrates duality: assuming that $\mathbf{R}$ is Anderson self-dual, without necessarily a gap in its homotopy, somehow peel off a connective piece from its coconnective dual, but for the present this is only a fantasy.

1.B. Description of contents. We start by giving an account of Anderson duality. The main point of this is to explain its limitations and to make explicit the way this works under change of ring spectra.

We recall the definition of Gorenstein ring spectra and Gorenstein duality. The Gorenstein condition only makes sense when we have a counterpart of a residue 
field. However Gorenstein duality makes sense more generally. Under orientability hypotheses the Gorenstein condition implies Gorenstein duality.

It is then straightforward to compare Gorenstein and Anderson duality, and we illustrate the usefulness of this in a number of cases.

Finally we finish by describing how to use Molien's theorem to predict the change of shift under passage to invariants 1

1.C. Conventions. We work in the homotopy category of modules over a ring spectrum. However we also need to know that there is a ring spectrum of endomorphisms of a module spectrum. For definiteness, we work with EKMM-spectra [EKMM97, but our results are not sensitive to models, so apply in other contexts with a homotopically meaningful symmetric monoidal smash product and internal Hom spectra.

Given a spectrum $X$, we write $\pi_{*} X=X_{*}$ for its coefficients, and we note that if $M$ is an $R$-module

$$
\pi_{*}(M)=[\mathbb{S}, M]_{*}=[R, M]_{*}^{R},
$$

where $\mathbb{S}$ is the sphere spectrum, and the superscript $R$ refers to working in the category of $R$-modules.

The basic context is that we are given a connective commutative ring spectrum. It is convenient to use the traditional convention of using lower case for connective covers, so we write $\mathbf{r}$ for the ring spectrum and $K=\pi_{0}(\mathbf{r})$. By killing homotopy groups in commutative ring spectra, we have a map $\epsilon: \mathbf{r} \longrightarrow H K$ of commutative ring spectra. In our main examples $K$ will be an $\mathbb{F}_{p}$ or a localization of $\mathbb{Z}$.

\section{Anderson DUALS}

The construction of Anderson duals is a two step process. For injective modules we apply Brown representability (to get the so-called Brown-Comenetz duals) and then we use cofibre sequences to obtain Anderson duals for modules of injective dimension 1. Since we are usually working over a field or a localization of a discrete valuation ring this covers many cases of interest. Unfortunately, the construction cannot be much generalized (at injective dimension 2 choice is involved, and at higher dimension the construction is often obstructed).

2.A. Construction of Brown-Comenetz duals. The basis for Anderson duality is that we can uniquely lift injective coefficient modules to module spectra. In general, we are in a situation where data as below is given.

\footnotetext{
${ }^{1}$ It is characteristic that Dave Benson not only wrote the book Ben93 from which we learnt this result but also illustrated it for us with numerous examples. We are grateful to him for his exposition, his vast range of interesting examples, and the delightful process of explanation.
} 
- We have maps of commutative ring spectra

$$
\mathbb{S} \longrightarrow S \longrightarrow R \text {. }
$$

Often we will take $S$ to equal the sphere spectrum $\mathbb{S}$ or $R$ itself, but it is useful to retain some flexibility.

- Additionally, we have a map of graded rings

$$
A_{*} \longrightarrow R_{*} \text {. }
$$

There is no requirement that $A_{*} \longrightarrow R_{*}$ is induced by a map of ring spectra. For example, we always have the unit map $A_{*}=\mathbb{Z} \longrightarrow R_{*}$ (in degree zero), and this is what plays a role in classical Brown-Comenetz duality [BC76].

The most common and important instance of the above occurs by taking $K=\pi_{0}(R)$, and declaring $A_{*}=K$ in degree zero, i.e. we consider the map

$$
K \longrightarrow R_{*} \text {. }
$$

The construction is that we take an injective $A_{*}$-module $J$ and consider the functor

$$
\begin{aligned}
R \text {-mod } \longrightarrow \mathrm{Ab}_{*} \\
\quad X \longmapsto \operatorname{Hom}_{A_{*}}\left(\pi_{*}(X), J\right) .
\end{aligned}
$$

Since $J$ is injective, this is a cohomology theory, and by Brown representability there is an $R$-module $J^{R}=J_{A_{*}}^{R}$ so that

$$
\left[X, J^{R}\right]_{*}^{R}=\operatorname{Hom}_{A_{*}}\left(\pi_{*}(X), J\right) .
$$

Slightly more generally, for an $R$-module $M$ we may define $J^{M}=J_{A_{*}}^{M}$ by the equation

$$
\left[X, J^{M}\right]_{*}^{R}=\operatorname{Hom}_{A_{*}}\left(\pi_{*}\left(X \otimes_{R} M\right), J\right) .
$$

One quickly checks that

$$
J^{M} \simeq \operatorname{Hom}_{R}\left(M, J^{R}\right),
$$

and we say $J^{M}$ is the Brown-Comenetz $J$-dual of $M$. Of course, $J^{R}$ is itself the Brown-Comenetz $J$-dual of $R$, and this is the case we will use the most.

2.B. Properties of Brown-Comenetz duals. We highlight four properties of the Brown-Comenetz dual.

(P0) (Homotopy groups) By construction,

$$
\pi_{*}\left(J_{A_{*}}^{M}\right)=\operatorname{Hom}_{A_{*}}\left(\pi_{*}(M), J\right) .
$$

(P1) (Eilenberg-MacLane spectra) If $R=H K$ is an Eilenberg-MacLane spectrum and $J$ is an ungraded injective $K$-module, then

$$
J_{K}^{H K}=H J
$$


Given a ring map $S \longrightarrow R$ and an $S$-module $N$ let us write

$$
N \Uparrow_{S}^{R}=\operatorname{Hom}_{S}(R, N)
$$

for the coextended module.

(P2) (Coextension of scalars I) Given $S \longrightarrow R$ and $A_{*} \longrightarrow S_{*} \longrightarrow R_{*}$ we have

$$
J_{A_{*}}^{R}=\left(J_{A_{*}}^{S}\right) \Uparrow_{S}^{R} .
$$

More generally, if $N$ is an $S$-module, we have

$$
J_{A_{*}}^{R \otimes_{S} N}=\left(J_{A_{*}}^{N}\right) \Uparrow_{S}^{R} .
$$

Proof: For an $R$-module $X$ we have

$$
\left[X, J_{A_{*}}^{R}\right]^{R}=\operatorname{Hom}_{A_{*}}\left(\pi_{*} X, J\right)=\left[X, J_{A_{*}}^{S}\right]^{S}=\left[X,\left(J_{A_{*}}^{S}\right) \Uparrow_{S}^{R}\right]^{R} .
$$

(P3) (Coextension of scalars II) Given $A_{*} \longrightarrow R_{*}$ we note that $J \Uparrow_{A_{*}}^{R_{*}}$ is injective and then we have

$$
\left(J \Uparrow_{A_{*}}^{R_{*}}\right)_{R_{*}}^{R} \simeq J_{A_{*}}^{R} .
$$

Proof: For an $R$-module $X$ we have

$$
\left[X,\left(J \Uparrow_{A_{*}}^{R_{*}}\right)_{R_{*}}^{R}\right]^{R}=\operatorname{Hom}_{R_{*}}\left(\pi_{*} X, J \Uparrow_{A_{*}}^{R_{*}}\right)=\operatorname{Hom}_{A_{*}}\left(\pi_{*} X, J\right)=\left[X, J_{A_{*}}^{R}\right]^{R} .
$$

Remark 2.1. Since coextension is a well-known construction, Property (P2) means that we only ever need the special case of the construction going from modules over coefficients $A_{*}$ to modules over an initial ring spectrum to whose coefficients $A_{*}$ maps. Property (P3) means that we only ever need the special case of the Anderson construction going from modules over coefficients to modules over the ring spectrum.

2.C. Construction of Anderson duals. Now we suppose given an $A_{*}$-module $L$ of injective dimension 1 with chosen resolution

$$
0 \longrightarrow L \longrightarrow J_{0} \longrightarrow J_{1} \longrightarrow 0 \text {. }
$$

We then define $L_{A_{*}}^{R}$ by the fibre sequence

$$
L_{A_{*}}^{R} \longrightarrow\left(J_{0}\right)_{A_{*}}^{R} \longrightarrow\left(J_{1}\right)_{A_{*}}^{R} .
$$

We note that the maps are determined by the defining properties and the original resolution, and it is not hard to show the spectrum is independent of the resolution. The classical example [And69] is that of $L=\mathbb{Z}=A_{*}$. 
As for the Brown-Comenetz case, we may also define the Anderson $L$-dual of an $R$-module $M$, either by replacing $R$ by $M$ in the above construction, or directly by taking $L_{A_{*}}^{M}=\operatorname{Hom}_{R}\left(M, L_{A_{*}}^{R}\right)$. Again, $L_{A_{*}}^{R}$ is itself the Anderson $L$-dual of $R$.

2.D. Properties of Anderson duals. The properties of the Anderson dual then follow from those of the Brown-Comenetz dual. We suppose that $L$ is an $A_{*}$-module of injective dimension $\leq 1$.

(P0) (Homotopy groups) There is a natural exact sequence

$$
0 \longrightarrow \operatorname{Ext}_{A_{*}}^{1}\left(\Sigma \pi_{*}(R), L\right) \longrightarrow \pi_{*}\left(L_{A_{*}}^{R}\right) \longrightarrow \operatorname{Hom}_{A_{*}}\left(\pi_{*}(R), L\right) \longrightarrow 0,
$$

and more generally one which computes the homotopy groups of the dual of any $R$-module $M$, using $\left[M, L_{A_{*}}^{R}\right]_{*}^{R}=\left[R, L_{A_{*}}^{M}\right]_{*}^{R}$ :

$$
0 \longrightarrow \operatorname{Ext}_{A_{*}}^{1}\left(\Sigma \pi_{*}(M), L\right) \longrightarrow\left[M, L_{A_{*}}^{R}\right]_{*}^{R} \longrightarrow \operatorname{Hom}_{A_{*}}\left(\pi_{*}(M), L\right) \longrightarrow 0 .
$$

(P1) (Eilenberg-MacLane spectra) If $R=H K$ is an Eilenberg-MacLane spectrum and $L$ is an ungraded $K$-module then

$$
L_{K}^{H K} \simeq H L .
$$

(P2) (Coextension of scalars I) Given $S \longrightarrow R$ and $A_{*} \longrightarrow S_{*} \longrightarrow R_{*}$, we have

$$
L_{A_{*}}^{R} \simeq\left(L_{A_{*}}^{S}\right) \Uparrow_{S}^{R}
$$

More generally, for an $S$-module $N$ we have

$$
L_{A_{*}}^{R \otimes_{S} N} \simeq\left(L_{A_{*}}^{N}\right) \Uparrow_{S}^{R} .
$$

The main case of interest is that if $A_{*}=\mathbb{Z}$ we need only use the classical Anderson dual of the sphere:

$$
\mathbb{Z}^{R} \simeq\left(I_{\mathbb{Z}}\right) \Uparrow_{\mathbb{S}}^{R}
$$

where $I_{\mathbb{Z}}=\mathbb{Z}^{\mathbb{S}}$ is the usual Brown-Comenetz dual of the sphere. Similar comments apply to localizations of $\mathbb{Z}$.

(P3) (Coextension of scalars II) Given $A_{*} \longrightarrow R_{*}$ we note that we may coextend the resolution of $L$ to show $L \Uparrow_{A_{*}}^{R_{*}}$ is of injective dimension $\leq 1$ and then we have

$$
\left(L \Uparrow_{A_{*}}^{R_{*}}\right)_{R_{*}}^{R} \simeq L_{A_{*}}^{R}
$$

\section{The Gorenstein COndition}

We recall the basic language and results of Gorenstein ring spectra from [DGI06]. Because of our applications, we will work with a map $\mathbf{r} \longrightarrow H K$, and we assume $\mathbf{r}$ is connective and denote $K=\pi_{0}(\mathbf{r})$. 
3.A. Cellularization. An r-module $X$ is said to be $H K$-cellular if it is in the localizing subcategory of $H K$ (i.e. it is constructed from $H K$ using triangles and coproducts). An $H K$-cellularization of an $\mathbf{r}$-module $M$ is a map $X \longrightarrow M$ so that $X$ is $H K$-cellular and the map is an $\operatorname{Hom}_{\mathbf{r}}(H K, \cdot)$-equivalence. The $H K$-cellularization is unique up to equivalence of $\mathbf{r}$-modules and we write $\operatorname{Cell}_{H K} M$ for it.

3.B. Morita theory. We say that the $H K$-cellularization of an r-module $M$ is effectively constructible if the natural evaluation map

$$
\operatorname{Hom}_{\mathbf{r}}(H K, M) \otimes_{\mathcal{E}} H K \longrightarrow M
$$

is $H K$-cellularization, where $\mathcal{E}=\operatorname{Hom}_{\mathbf{r}}(H K, H K)$.

We recall that $H K$ is proxy-small if $H K$ finitely builds a small object $\widetilde{H K}$ which generates the same localizing subcategory of $R$-modules. The proxy-smallness condition is very mild, but in most of our applications here we will be in the situation that $H K$ is actually small so that we may take $\widetilde{H K}=H K$.

The fact [DGI06, 4.9] we use is that if $H K$ is proxy-small as an r-module, then every r-module has an effectively constructible $H K$-cellularization.

3.C. The Gorenstein condition. The basic definition was given for ring spectra in DGI06].

Definition 3.1. We say that $\mathbf{r} \longrightarrow H K$ is Gorenstein of shift a if $\operatorname{Hom}_{\mathbf{r}}(H K, \mathbf{r}) \simeq$ $\Sigma^{a} H K$.

If we suppose $\mathbf{r} \longrightarrow H K$ is Gorenstein of shift $a$, we may wonder how this compares to other modules $\mathbb{I}$ lifting $H K$ in the sense that $\operatorname{Hom}_{\mathbf{r}}\left(H K, \Sigma^{a} \mathbb{I}\right) \simeq \Sigma^{a} H K$. For example the Anderson dual spectrum $\mathbb{I}=K_{K}^{\mathbf{r}}=: K^{\mathbf{r}}$ as in Subsections 2.C and 2.D qualifies as the 'trivial' lift, and in Section 4 and beyond, we will restrict attention to that case. For now just assume that $\mathbb{I}$ is an $H K$-cellular $\mathbf{r}$-module with the required lifting property, and note that the notions of Gorenstein orientability and duality below implicitly depend on $\mathbb{I}$.

If $\mathbf{r} \longrightarrow H K$ is Gorenstein, we have

$$
\operatorname{Hom}_{\mathbf{r}}(H K, \mathbf{r}) \simeq \Sigma^{a} H K \simeq \operatorname{Hom}_{\mathbf{r}}\left(H K, \Sigma^{a} \mathbb{I}\right) .
$$

We note that the ring spectrum $\mathcal{E}=\operatorname{Hom}_{\mathbf{r}}(H K, H K)$ acts on the right of both of these modules.

Definition 3.2. We say that $\mathbf{r}$ is orientably Gorenstein if the equivalence

$$
\operatorname{Hom}_{\mathbf{r}}(H K, \mathbf{r}) \simeq \operatorname{Hom}_{\mathbf{r}}\left(H K, \Sigma^{a} \mathbb{I}\right)
$$

is an equivalence of right $\mathcal{E}$-modules. 
3.D. Gorenstein duality. If $\mathbf{r} \longrightarrow H K$ is orientably Gorenstein and $H K$ is proxysmall, we may apply the equivalence from Morita theory (Subsection 3.B) to deduce

$$
\mathrm{Cell}_{H K} \mathbf{r} \simeq \Sigma^{a} \mathrm{Cell}_{H K} \mathbb{I} .
$$

For example the Anderson dual $\mathbb{I}=K^{\mathbf{r}}$ is bounded above, with each homotopy group a $K$-module, and hence it is already $H K$-cellular, so that $\operatorname{Cell}_{H K} K^{\mathbf{r}}=K^{\mathbf{r}}$. The above condition translates to an equivalence

$$
\text { Cell }_{H K} \mathbf{r} \simeq \Sigma^{a} K^{\mathbf{r}} .
$$

Definition 3.3. We say that $\mathbf{r} \longrightarrow H K$ has torsion Gorenstein duality of shift $a$ if

$$
\text { Cell }_{H K} \mathbf{r} \simeq \Sigma^{a} \mathbb{I} \text {. }
$$

Rather often this is used by completing both sides, which is to say applying the functor

$$
(\cdot)_{H K}^{\wedge}=\operatorname{Hom}_{\mathbf{r}}\left(\operatorname{Cell}_{H K} \mathbf{r}, \cdot\right) .
$$

Definition 3.4. We say that $\mathbf{r} \longrightarrow H K$ has complete Gorenstein duality of shift a if

$$
\mathbf{r}_{H K}^{\wedge} \simeq \Sigma^{a} \mathbb{I}_{H K}^{\wedge}
$$

Remark 3.5. Since $\mathbf{r}$ is connective it is the inverse limit of its Postnikov sections and hence $H K$-complete, i.e. $\mathbf{r}_{H K}^{\wedge} \simeq \mathbf{r}$ and the condition simplifies to the statement

$$
\mathbf{r} \simeq \Sigma^{a} \mathbb{I}_{H K}^{\wedge}
$$

In fact the two Gorenstein duality conditions are equivalent, so that when no emphasis is necessary we refer simply to 'Gorenstein duality'.

Lemma 3.6. The torsion and complete Gorenstein duality statements are equivalent.

Proof: Since the map Cell $_{H K} \mathbf{r} \longrightarrow \mathbf{r}$ is an $H K$-cellular equivalence, it is clear that the torsion duality implies complete duality by taking completions, since

$$
\operatorname{Hom}_{\mathbf{r}}\left(\operatorname{Cell}_{H K} \mathbf{r}, \operatorname{Cell}_{H K} \mathbf{r}\right) \simeq \operatorname{Hom}_{\mathbf{r}}\left(\operatorname{Cell}_{H K} \mathbf{r}, \mathbf{r}\right) \simeq \mathbf{r}_{H K}^{\wedge}
$$

To recover the torsion duality from complete duality, we use $H K$-cellularizations as follows.

In fact completion is a cellular equivalence rather generally. We consider the completion map

$$
M=\operatorname{Hom}_{\mathbf{r}}(\mathbf{r}, M) \longrightarrow \operatorname{Hom}_{\mathbf{r}}\left(\operatorname{Cell}_{H K} \mathbf{r}, M\right)
$$

and apply $\operatorname{Hom}_{\mathbf{r}}(H K, \cdot)$ to get

$$
\operatorname{Hom}_{\mathbf{r}}\left(H K \otimes_{\mathbf{r}} \mathbf{r}, M\right) \longrightarrow \operatorname{Hom}_{\mathbf{r}}\left(H K \otimes_{\mathbf{r}} \operatorname{Cell}_{H K} \mathbf{r}, M\right) .
$$


We observe this is an equivalence; indeed, since $H K$ is $H K$-cellular and $H K$ cellularization is smashing, the map

$$
H K \otimes_{\mathbf{r}} \operatorname{Cell}_{H K} \mathbf{r} \longrightarrow H K \otimes_{\mathbf{r}} \mathbf{r}
$$

is an equivalence. Thus

$$
\operatorname{Cell}_{H K} M \simeq \operatorname{Hom}_{\mathbf{r}}\left(\operatorname{Cell}_{H K} \mathbf{r}, M\right) \otimes_{\mathbf{r}} \operatorname{Cell}_{H K} \mathbf{r} \simeq \operatorname{Cell}_{H K}\left(M_{H K}^{\wedge}\right)
$$

as required.

3.E. Gorenstein duality relative to $\mathbb{F}_{p}$. We consider the statement of Gorenstein duality for $\mathbf{r} \longrightarrow H \mathbb{F}_{p}$ when $K=\pi_{0}(\mathbf{r}) \cong \mathbb{Z}$ (or equally when $K=\mathbb{Z}_{(p)}, \mathbb{Z}_{p}^{\wedge}$ ), referring to the discussion in the previous subsection for comparison.

As before we start by assuming $\mathbf{r} \longrightarrow H \mathbb{F}_{p}$ is Gorenstein of shift $a$, and note that this gives an equivalence

$$
\operatorname{Hom}_{\mathbf{r}}\left(H \mathbb{F}_{p}, \mathbf{r}\right) \simeq \Sigma^{a} H \mathbb{F}_{p} \simeq \operatorname{Hom}_{\mathbf{r}}\left(H \mathbb{F}_{p}, \Sigma^{a+1} K^{\mathbf{r}}\right) .
$$

The difference from the case relative to $H K$ is that

$$
\operatorname{Cell}_{H \mathbb{F}_{p}}\left(K^{\mathbf{r}}\right) \simeq \Sigma^{-1}\left(\mathbb{Z} / p^{\infty}\right)^{\mathbf{r}}
$$

The appropriate definition is then clear.

Definition 3.7. When $K=\pi_{0}(\mathbf{r})=\mathbb{Z}, \mathbb{Z}_{(p)}, \mathbb{Z}_{p}^{\wedge}$, we say that $\mathbf{r} \longrightarrow H \mathbb{F}_{p}$ has Gorenstein duality of shift $a$ if

$$
\operatorname{Cell}_{H \mathbb{F}_{p}} \mathbf{r} \simeq \Sigma^{a}\left(\mathbb{Z} / p^{\infty}\right)^{\mathbf{r}}
$$

As before, if $\mathbf{r} \longrightarrow H \mathbb{F}_{p}$ is proxy regular and there is a unique action of $\operatorname{Hom}_{\mathbf{r}}\left(\mathbb{F}_{p}, \mathbb{F}_{p}\right)$ on $\mathbb{F}_{p}$, then Gorenstein implies Gorenstein duality.

In the context where both make sense, we show in the next subsection that this Gorenstein duality is equivalent to the duality of Mahowald-Rezk [MR99].

3.F. Mahowald-Rezk duality. Mahowald and Rezk [MR99] consider the class of $f p$-spectra (connective, $p$-complete and whose $\bmod p$ homology is a finitely presented comodule over the Steenrod algebra). The type of a $p$-local finite complex $F$ is defined by

$$
\operatorname{type}(F)=\min \left\{n \mid K(n)_{*} F \neq 0\right\},
$$

where $K(n)$ is the $n$th Morava $K$-theory at $p$. The $f p$-type of an fp-spectrum $X$ is defined by

fp-type $(X)=\min \left\{\operatorname{type}(F)-1 \mid F\right.$ is a finite complex and $\pi_{*}(X \wedge F)$ is a finite group $\}$.

For example, $k o$ and $k u$ are fp-spectra of fp-type 1 , and tmf is an fp-spectrum of fp-type 2. 
If $\mathbf{r}$ is a ring spectrum of fp-type $n$, such that its mod-p homology is self-dual in a suitable sense, then Mahowald and Rezk show that there is a duality equivalence

$$
\left(\mathbb{Z} / p^{\infty}\right)^{C_{n}^{f} \mathbf{r}} \simeq \Sigma^{c} \mathbf{r}
$$

This is satisfied in a number of cases, including $k o, k u$, and tmf [MR99, Proposition 9.2, Corollary 9.3]. Here $C_{n}^{f}$ is the $n$th finite chromatic cellularization (i.e., the cellularization with respect to a finite type $n+1$ complex $F$ ). A more specific construction proceeds by constructing a cofinal inverse system of generalized Moore spectra $S^{0} / I=S^{0} / v_{0}^{i_{0}}, v_{1}^{i_{1}}, \ldots, v_{n}^{i_{n}}$ and then taking

$$
C_{n}^{f} X=\underset{\rightarrow}{\operatorname{holim}} F\left(S^{0} / I, X\right) .
$$

Lemma 3.8. If $\mathbf{r}$ is an fp-spectrum of fp-type $n$ then there is a natural equivalence $C_{n}^{f} M \simeq \operatorname{Cell}_{H \mathbb{F}_{p}} M$ for $\mathbf{r}$-modules $M$.

Proof: The proof consists of two steps: identify $C_{n}^{f} M$ with the cellularisation in $\mathbf{r}$ modules $\operatorname{Cell}_{F \wedge \mathbf{r}}$, and then show that the localising subcategories $\left\langle H \mathbb{F}_{p}\right\rangle$ and $\langle F \wedge \mathbf{r}\rangle$ of $\mathbf{r}$-modules, generated by $H \mathbb{F}_{p}$ and $F \wedge \mathbf{r}$, respectively, are equal.

For the first step, we check that $\operatorname{Cell}_{F \wedge \mathbf{r}} M$ has the required universal property. Let $M[1 / F \wedge \mathbf{r}]$ denote the cofibre of the natural map $\operatorname{Cell}_{F \wedge \mathbf{r}} M \rightarrow M$; then spectrum maps from $F$ to $M[1 / F \wedge \mathbf{r}]$ are the same thing as $\mathbf{r}$-module maps from $F \wedge \mathbf{r}$ to it, but by construction those are all null. Next, we need to know that the spectrum underlying $\operatorname{Cell}_{F \wedge \mathbf{r}} M$ is in the localising subcategory of spectra generated by $F$. Since colimits commute with smash product, this follows since the $\mathbf{r}$-module $\operatorname{Cell}_{F \wedge \mathbf{r}} M$ is in the localising subcategory of $\mathbf{r}$-modules generated by $F \wedge \mathbf{r}$.

For the second step, the key property is that $F \wedge \mathbf{r}$ is a finite wedge of copies of $H \mathbb{F}_{p}$ by [MR99, Proposition 3.2]. Hence, $F \wedge \mathbf{r}$ is in the localising subcategory $\left\langle H \mathbb{F}_{p}\right\rangle$ (argue by induction that if $\pi_{*}(M)$ is a finite dimensional vector space it is finitely built by $H \mathbb{F}_{p}$; for the inductive step, if $M$ has bottom homotopy in degree 0 , killing homotopy groups in $\mathbf{r}$-modules, gives a map $M \longrightarrow H \mathbb{F}_{p}$ non-zero in $\pi_{0}$ ). Conversely $H \mathbb{F}_{p}$ is in $\langle F \wedge \mathbf{r}\rangle$ (if $M$ is a module which is a finite wedge of copies of $H \mathbb{F}_{p}$ as a spectrum, then we can construct a map from a finite wedge of copies of $F \wedge \mathbf{r}$ that is surjective on the bottom homotopy; since $F \wedge \mathbf{r}$ is small, repeating this and passing to direct limits, we may kill all homotopy. To construct the map, note that for any chosen element of $\pi_{0}$ there is a map $F \longrightarrow M$ which maps onto it, and we extend it to an $\mathbf{r}$-module map $\mathbf{r} \wedge F \longrightarrow M$ ).

Accordingly, the Mahowald-Rezk duality statement reads

$$
\left(\mathbb{Z} / p^{\infty}\right)^{\operatorname{Cell}_{H \mathbb{F}} \mathbf{r}} \simeq \Sigma^{c} \mathbf{r}
$$


Assuming the homotopy groups of $\mathbf{r}$ are profinitely complete, we may dualize to find

$$
\operatorname{Cell}_{H \mathbb{F}_{p}} \mathbf{r} \simeq \Sigma^{-c}\left(\mathbb{Z} / p^{\infty}\right)^{\mathbf{r}}
$$

When $\pi_{0}(\mathbf{r})=\mathbb{Z}_{p}^{\wedge}$, this is precisely the statement that $\mathbf{r} \longrightarrow H \mathbb{F}_{p}$ is Gorenstein of shift $-c$. Summarising, the above gives the following conclusion.

Lemma 3.9. If $\mathbf{r}$ is an fp-spectrum of fp-type $n$, whose homotopy groups are $p$ complete, then $\mathbf{r} \rightarrow H \mathbb{F}_{p}$ is Gorenstein of shift $-c$ if and only if $\mathbf{r}$ is Mahowald-Rezk self-dual of shift $c$.

\section{Gorenstein DuAlity And Anderson Self-DUAlity}

In this section we explain that Gorenstein duality for a connective ring spectrum gives an Anderson self-duality for the associated non-connective spectrum.

We note that Anderson duality exchanges connective and coconnective spectra, so we cannot expect to have self-duality for connective spectra. Similarly, periodic spectra often fail to have residue fields, so that the Gorenstein condition usually makes no sense for them. Accordingly each approach plays an essential role.

4.A. Nullifying $H K$. From our connective ring spectrum $\mathbf{r}$ and the map $\mathbf{r} \longrightarrow H K$ obtained by killing higher homotopy groups we may form a cofibre sequence

$$
\mathrm{Cell}_{H K} \mathbf{r} \longrightarrow \mathbf{r} \longrightarrow \mathbf{r}[1 / H K]
$$

where $\mathbf{r} \longrightarrow \mathbf{r}[1 / H K]$ is the initial map to a spectrum with no maps from $H K$. We take $\mathbf{R}=\mathbf{r}[1 / H K]$, and it is a commutative ring spectrum since $\mathbf{r}$ is.

4.B. Anderson self-duality from Gorenstein duality. We are ready to bring the threads together. The most interesting implication is that Anderson self-duality follows from Gorenstein duality.

Proposition 4.1. If $\mathbf{r} \longrightarrow H K$ has Gorenstein duality of shift a then $\mathbf{r}[1 / H K]$ has Anderson self-duality with shift $a+1$ in the sense that

$$
K^{\mathbf{r}[1 / H K]} \simeq \Sigma^{-a-1} \mathbf{r}[1 / H K] .
$$

Furthermore,

(i) $K^{\mathbf{r}} \simeq \Sigma^{-a} \mathrm{Cell}_{H K} \mathbf{r}$.

(ii) The map $\epsilon:$ Cell $_{H K} \mathbf{r} \longrightarrow \mathbf{r}$ is self dual: if we apply $\operatorname{Hom}_{\mathbf{r}}\left(\cdot, K^{\mathbf{r}}\right)$ to $\epsilon$, we obtain the ath desuspension of $\epsilon$.

Remark 4.2. Note that the Anderson self-duality statement makes it natural to write the suspension on the side of the ring

$$
K^{\mathbf{R}} \simeq \Sigma^{-a-1} \mathbf{R}
$$


since it says the Anderson dual is a shift of the original ring. The Gorenstein duality statement makes it natural to put the suspension on the side of the Anderson dual

$$
\text { Cell }_{H K} \mathbf{r} \simeq \Sigma^{a} K^{\mathbf{r}},
$$

since it says the cellularization is a shift of a naive expectation.

Of course it is easy to pass between the two, but the first convention focuses on a shift (viz $-a-1)$ that is usually positive whereas the second convention focuses on a shift (viz $a$ ) that is usually negative.

Proof: Part (i) is a restatement of Gorenstein duality, and the Anderson self-duality is an immediate consequence of Part (ii).

It remains only to prove that the map in (ii) is self dual. However we note that maps $\epsilon:$ Cell $_{H K} \mathbf{r} \longrightarrow \mathbf{r}$ are easily classified since $\operatorname{Hom}_{\mathbf{r}}\left(\operatorname{Cell}_{H K} \mathbf{r}, \mathbf{r}\right) \simeq \mathbf{r}$ with $\pi_{0}(\mathbf{r})=$ $K$.

To see that the dual of $\epsilon$ is as required, let

$$
\rho: \operatorname{Cell}_{H K} \mathbf{r} \stackrel{\simeq}{\longrightarrow} \Sigma^{a} K^{\mathbf{r}}
$$

be the given equivalence. Since $\rho$ is an equivalence we may use $\operatorname{Hom}_{\mathbf{r}}\left(\cdot, \Sigma^{-a} \operatorname{Cell}_{H K} \mathbf{r}\right)$ as the dualization. Then $\epsilon$ dualizes to

$$
\begin{aligned}
\epsilon^{*}: \Sigma^{-a} \operatorname{Cell}_{H K} \mathbf{r} \simeq & \operatorname{Hom}_{\mathbf{r}}\left(\mathbf{r}, \Sigma^{-a} \operatorname{Cell}_{H K} \mathbf{r}\right) \longrightarrow \\
& \longrightarrow \operatorname{Hom}_{\mathbf{r}}\left(\operatorname{Cell}_{H K} \mathbf{r}, \Sigma^{-a} \operatorname{Cell}_{H K} \mathbf{r}\right) \simeq \Sigma^{-a} \mathbf{r}_{H K}^{\wedge} \simeq \Sigma^{-a} \mathbf{r},
\end{aligned}
$$

where the last equivalence is because $\mathbf{r}$ is connective (see Remark 3.5). It is easy to see this has the universal property of cellularization and is therefore the suspension of $\epsilon$.

On the other hand, if we have Anderson self-duality in the sense that

$$
\Sigma^{a+1} K^{\mathbf{r}[1 / H K]} \simeq \mathbf{r}[1 / H K]
$$

then it is not automatic that $\mathbf{r}$ has Gorenstein duality without additional connectivity statements (for example Meier [Mei16] shows $\operatorname{Tmf}_{1}(23)$ is Anderson self-dual, with $a=0$, whereas one can see by considering complex modular forms with level 23 structure that its connective cover does not enjoy Gorenstein duality).

Lemma 4.3. Suppose that

$$
K^{\mathbf{r}[1 / H K]} \simeq \Sigma^{-a-1} \mathbf{r}[1 / H K]
$$

with $a \leq-2$.

If $\pi_{i}\left(\operatorname{Cell}_{H K} \mathbf{r}\right)=0$ for $i \geq a+1$, and $\pi_{a}\left(\operatorname{Cell}_{H K} \mathbf{r}\right)$ is projective over $K$, then $\mathbf{r}$ has Gorenstein duality of shift a. 
Proof: We apply $\operatorname{Hom}\left(\cdot, K^{\mathbf{r}}\right)$ to the cofibre sequence

$$
\mathrm{Cell}_{H K} \mathbf{r} \longrightarrow \mathbf{r} \longrightarrow \mathbf{r}[1 / H K]
$$

to obtain

$$
\operatorname{Hom}_{\mathbf{r}}\left(\operatorname{Cell}_{H K} \mathbf{r}, K^{\mathbf{r}}\right) \longleftarrow K^{\mathbf{r}} \longleftarrow \Sigma^{-a-1} \mathbf{r}[1 / H K] .
$$

Suspending $a$ times and taking mapping cones, we obtain the cofibre sequence

$$
\mathbf{r}[1 / H K] \longleftarrow \Sigma^{a} \operatorname{Hom}_{\mathbf{r}}\left(\operatorname{Cell}_{H K} \mathbf{r}, K^{\mathbf{r}}\right) \longleftarrow \Sigma^{a} K^{\mathbf{r}},
$$

and we want to check that this is equivalent to the original (1).

From the hypotheses, $\pi_{t}\left(\Sigma^{a} \operatorname{Hom}_{\mathbf{r}}\left(\operatorname{Cell}_{H K} \mathbf{r}, K^{\mathbf{r}}\right)\right)$ is zero for $t \leq-1$. Indeed, from the Anderson dual Property (P0), this homotopy group sits in an exact sequence

$$
\begin{aligned}
0 \longrightarrow \operatorname{Ext}_{K}^{1}\left(\pi_{-t+a-1} \operatorname{Cell}_{H K} \mathbf{r}, K\right) & \longrightarrow \pi_{t}\left(\Sigma^{a} \operatorname{Hom}_{\mathbf{r}}\left(\operatorname{Cell}_{H K} \mathbf{r}, K^{\mathbf{r}}\right)\right) \\
& \longrightarrow \operatorname{Hom}_{K}\left(\pi_{-t+a}\left(\operatorname{Cell}_{H K} \mathbf{r}\right), K\right) \longrightarrow 0,
\end{aligned}
$$

and for $t \leq-1$, both the Hom and Ext term vanish. Hence

$\mathbf{r} \simeq(\mathbf{r}[1 / H K])[a+2, \infty) \simeq\left(\Sigma^{a} \operatorname{Hom}_{\mathbf{r}}\left(\operatorname{Cell}_{H K} \mathbf{r}, K^{\mathbf{r}}\right)\right)[a+2, \infty) \simeq \Sigma^{a} \operatorname{Hom}_{\mathbf{r}}\left(\operatorname{Cell}_{H K} \mathbf{r}, K^{\mathbf{r}}\right) ;$

the first and second equivalence are because $\left(\operatorname{Cell}_{H K} \mathbf{r}\right)[a+2, \infty)$ and $\left(\Sigma^{a} K^{\mathbf{r}}\right)[a+2, \infty)$ respectively are contractible.

Thus the middle term of the sequence (2) is $\mathbf{r}$; it remains to check that its map to $\mathbf{r}[1 / H K]$ satisfies the requisite universal property. This follows since the fibre $\Sigma^{a} K^{\mathbf{r}}$ is clearly $H K$-cellular. We conclude that $\operatorname{Cell}_{H K} \mathbf{r} \simeq \Sigma^{a} K^{\mathbf{r}}$ as required.

\section{EXAmples With POLYNOMial OR HYPERSURFACE COEFFICIENT RINGS}

There are quite a number of examples that are algebraically very simple, so that we can apply our results without additional work, and we discuss a selection of those here.

5.A. The Cech complex. When the coefficient ring is simple, we have very algebraic models of the cellularization $\operatorname{Cell}_{H K} M$ and $M[1 / H K]$. We briefly recall the construction here (see GM95] for more details).

Suppose that $\mathfrak{n}=\left(x_{1}, \ldots, x_{r}\right)$ is an ideal in the coefficient ring $\mathbf{r}_{*}$. There is an elementary construction of the Cech spectrum $\check{C}_{\mathfrak{n}} \mathbf{r}$ as follows. First we form the stable Koszul complex

$$
\Gamma_{\mathfrak{n}} \mathbf{r}=\Gamma_{\left(x_{1}\right)} \mathbf{r} \otimes_{\mathbf{r}} \cdots \otimes_{\mathbf{r}} \Gamma_{\left(x_{r}\right)} \mathbf{r}
$$

where $\Gamma_{(x)} \mathbf{r}=$ fibre $(\mathbf{r} \longrightarrow \mathbf{r}[1 / x])$. We note that the homotopy type does not depend on the particular generators $x_{i}$. Indeed, it is obvious that replacing generators $x_{i}$ by powers has no effect, and it is not hard to see that $\Gamma_{\mathfrak{n}}$ only depends on the radical of the ideal $\mathfrak{n}$. 
Now define $\check{C}_{\mathfrak{n}} \mathbf{r}$ by the fibre sequence

$$
\Gamma_{\mathfrak{n}} \mathbf{r} \longrightarrow \mathbf{r} \longrightarrow \check{C}_{\mathfrak{n}} \mathbf{r}
$$

It is easy to check that $\check{C}_{\mathfrak{n}} \mathbf{r}$ is a commutative ring up to homotopy, but it can also be constructed [GM95] as the nullification

$$
\check{C}_{\mathfrak{n}} \mathbf{r} \simeq \mathbf{r}\left[\frac{1}{(\mathbf{r} / \mathbf{x})}\right]
$$

where

$$
\mathbf{r} / \mathbf{x}=\mathbf{r} / x_{1} \otimes_{\mathbf{r}} \cdots \otimes_{\mathbf{r}} \mathbf{r} / x_{n}
$$

is the unstable Koszul complex. It follows that $\check{C}_{\mathfrak{n}} \mathbf{r}$ admits the structure of a commutative ring.

The case we have in mind is that $\mathbf{r}$ is connective with $\mathbf{r}_{*}$ Noetherian and

$$
\mathfrak{n}=\operatorname{ker}\left(\mathbf{r}_{*} \longrightarrow \mathbf{r}_{0}=K\right) \text {. }
$$

The relevance is clear from a lemma.

Lemma 5.1. Suppose $\mathbf{r}_{*}$ is a polynomial ring over $K$ or a hypersurface (i.e. $\mathbf{r}_{*}=$ $K\left[x_{1}, \ldots, x_{n}\right] /(f)$ with $f$ of positive degree). The $\mathbf{r}$-module $H K$ is proxy-small. For an $\mathbf{r}$-module $M$ we have equivalences

$$
\operatorname{Cell}_{H K} M \simeq \Gamma_{\mathfrak{n}} M \text { and } \check{C}_{\mathfrak{n}} M \simeq M\left[\frac{1}{H K}\right]
$$

Proof: We will show that $H K$ finitely builds $\widetilde{H K}=\mathbf{r} / \mathbf{x}$ and $\widetilde{H K}$ builds $H K$. This shows that $\widetilde{H K}$ is a witness for the proxy-smallness of $H K$ and in particular shows that $H K$ and $\widetilde{H K}$ generate the same localizing subcategory.

If $\mathbf{r}_{*}$ is a polynomial ring then $H K$ is itself small: we take $\widetilde{H K}=\mathbf{r} / \mathbf{x}$. We have a map $\mathbf{r} \longrightarrow \widehat{H K}$ and calculation immediately shows it is an isomorphism in $\pi_{0}$ so that $H K \simeq \widetilde{H K}$.

If $\mathbf{r}_{*}=K\left[x_{1}, \ldots, x_{r}\right] /(f)$ with $f$ of degree $s$ then we take $\widetilde{H K}=\mathbf{r} / \mathbf{x}$ and calculate $\pi_{*}(\widetilde{H K})=K[\phi] /\left(\phi^{2}\right)$, where $\phi$ is of degree $s+1$. We need only observe this is additively the homology of the short cochain complex

$$
\Sigma^{s} \mathbf{r}_{*} \stackrel{f}{\longrightarrow} \mathbf{r}_{*} \text {. }
$$

To see this, consider the polynomial ring $P=K\left[x_{1}, \ldots, x_{r}\right]$ and form the Koszul complex $K P$ for the elements $f, x_{1}, \cdots, x_{r}$. If we view $K P$ as a multicomplex and take homology in the order stated, it is the homology of the displayed complex. If we take homology in the order $x_{1}, \ldots, x_{r}, f$ then it is evidently $K[\phi] /\left(\phi^{2}\right)$. Killing homotopy groups in r-modules gives a cofibre sequence $\Sigma^{s+1} H K \longrightarrow \widehat{H K} \longrightarrow H K$ showing that $H K$ finitely builds $\widehat{H K}$. 
Similarly we may construct $H K$ from $\widetilde{H K}$ by a process of killing homotopy groups, but now using $\widetilde{H K}$ only. More precisely, we take $H K^{0}=\widetilde{H K}$ and iteratively construct $H K^{t+1}$ using a cofibre sequence

$$
\Sigma^{t(s+2)-1} \widetilde{H K} \longrightarrow H K^{t} \longrightarrow H K^{t+1}
$$

where $\pi_{*}\left(H K^{t}\right)$ is zero except in degrees 0 and $t(s+2)-1$ where it is $K$. The attaching map is chosen to be an isomorphism in degree $t(s+2)-1$. We see that $H K^{\infty}=\underset{\rightarrow}{\operatorname{holim}} H K^{t}$ is an Eilenberg-MacLane spectrum, and the map

$$
\mathbf{r} \longrightarrow \widetilde{H K}=H K^{0} \longrightarrow \underset{\rightarrow}{\operatorname{holim}} H K^{t}
$$

is an isomorphism in $\pi_{0}$ showing that $H K \simeq \underset{\rightarrow}{\operatorname{holim}} H K^{t}$ as r-modules.

5.B. The algebraic context. As usual we have a connective ring spectrum $\mathbf{r}$ with $\pi_{0}(\mathbf{r})=K$. We assume that $K$ is a localization or a completion of a number ring (usually $\mathbb{Z}$ ), that $\mathbf{r}_{*}$ is in even degrees, free over $K$ and of Krull dimension 2 and is either polynomial or a hypersurface ring. Some examples are tabulated in Subsection 5.D below.

In fact we suppose

$$
\mathbf{r}_{*}=K[x, y, z] /(f) \text { with }|x|=i,|y|=j,|z|=k,|f|=d .
$$

The case of a polynomial ring is a little simpler, but in any case it is covered by taking $f=z$.

Thus $\mathbf{r}_{*}$ is a relative complete intersection, and $\mathbf{r}_{*}$ is Gorenstein (and accordingly $\mathbf{r}$ is itself Gorenstein). Indeed, it is easy to calculate local cohomology (the cohomology of the stable Koszul complex), directly or by local duality to see

$$
H_{\mathfrak{n}}^{*}\left(\mathbf{r}_{*}\right)=H_{\mathfrak{n}}^{2}\left(\mathbf{r}_{*}\right)=\Sigma^{a+2} \mathbf{r}_{*}^{\vee}
$$

where $\mathbf{r}_{*}^{\vee}=\operatorname{Hom}_{K}\left(\mathbf{r}_{*}, K\right)$ and $a=d-(i+j+k)-2$. Since this is in a single cohomological degree the spectral sequence [Gre93, Theorem 4.1] for calculating homotopy collapses to give

$$
\pi_{*}\left(\Gamma_{\mathfrak{n}} \mathbf{r}\right)=\Sigma^{a} \mathbf{r}_{*}^{\vee}=\pi_{*}\left(\Sigma^{a} K^{\mathbf{r}}\right) .
$$

Assuming $a \leq-2$, the map $\Gamma_{\mathfrak{n}} \mathbf{r} \longrightarrow \mathbf{r}$ is zero in homotopy and the cofibre sequence

$$
\Gamma_{\mathfrak{n}} \mathbf{r} \longrightarrow \mathbf{r} \longrightarrow \check{C}_{\mathfrak{n}} \mathbf{r}
$$

gives an isomorphism

$$
\pi_{*}\left(\check{C}_{\mathfrak{n}} \mathbf{r}\right)=\mathbf{r}_{*} \oplus \Sigma^{a+1} \mathbf{r}_{*}^{\vee}
$$

since $a$ is even, and $\mathbf{r}_{*}$ is in even degrees, this is an isomorphism of $\mathbf{r}_{*}$-modules. 
From the algebraic isomorphism

$$
\pi_{*}\left(\Gamma_{\mathfrak{n}} \mathbf{r}\right)=\pi_{*}\left(\Sigma^{a} K^{\mathbf{r}}\right)
$$

we choose an isomorphism $\pi_{a}\left(\Gamma_{\mathfrak{n}} \mathbf{r}\right) \stackrel{\cong}{\longrightarrow} \pi_{a}\left(\Sigma^{a} K^{\mathbf{r}}\right)$, and since the homotopy of $\Gamma_{\mathfrak{n}} \mathbf{r}$ is free over $K$, the defining property of the Anderson dual gives a residue map

$$
\rho: \Gamma_{\mathfrak{n}} \mathbf{r} \longrightarrow \Sigma^{a} K^{\mathbf{r}} \text {. }
$$

To see that $\rho$ is an equivalence we note that both domain and codomain are $H K$ cellular, and hence it is enough to show it induces an equivalence

$$
\Sigma^{a} H K=\operatorname{Hom}_{\mathbf{r}}\left(H K, \Gamma_{\mathfrak{n}} \mathbf{r}\right) \stackrel{\rho_{*}}{\longrightarrow} \operatorname{Hom}_{\mathbf{r}}\left(H K, \Sigma^{a} K^{\mathbf{r}}\right)=\Sigma^{a} H K .
$$

We note that this shows that $\mathbf{r}$ has Gorenstein duality, since the spectrum $H K$ has a unique $\mathcal{E}$-module structure by connectivity.

5.C. A family of examples. Our examples come from derived algebraic geometry. We concentrate on the case of topological modular forms with level structure for definiteness. We begin with the compactified moduli stack $\overline{\mathfrak{M}}=\overline{\mathfrak{M}}_{\text {ell }}(\Gamma)$ of elliptic curves with level $\Gamma$ structure, on which we have the Goerss-Hopkins-Miller sheaf $\mathcal{O}^{\text {top }}$ of $E_{\infty}$-ring spectra (see HL16] for the log-étale refinement appropriate for level structures), and then take

$$
\operatorname{Tmf}(\Gamma)=\Gamma\left(\overline{\mathfrak{M}}_{\text {ell }}(\Gamma), \mathcal{O}^{\text {top }}\right) .
$$

The homotopy groups of this are calculated through a spectral sequence

$$
H^{s}\left(\overline{\mathfrak{M}}_{e l l}(\Gamma) ; \omega^{\otimes t}\right) \Rightarrow \operatorname{Tmf}(\Gamma)_{2 t-s}
$$

where $\omega$ denotes the sheaf of invariant differentials on $\overline{\mathfrak{M}}_{\text {ell }}(\Gamma)$. Consider those level structures for which $\overline{\mathfrak{M}}_{\text {ell }}(\Gamma)$ is representable. Then $\overline{\mathfrak{M}}_{\text {ell }}(\Gamma)$ is in fact a curve, and thus the spectral sequence collapses to give

$$
\operatorname{Tmf}(\Gamma)_{2 t}=H^{0}\left(\overline{\mathfrak{M}}_{\text {ell }}(\Gamma) ; \omega^{\otimes t}\right)
$$

and

$$
\operatorname{Tmf}(\Gamma)_{2 t-1}=H^{1}\left(\overline{\mathfrak{M}}_{\text {ell }}(\Gamma) ; \omega^{\otimes t}\right) .
$$

Assume that $H^{1}\left(\overline{\mathfrak{M}}_{\text {ell }}(\Gamma) ; \omega^{\otimes t}\right)$ is zero for all $t \geq 0$ (which happens in many cases), so that the contribution from $H^{1}$ is entirely in negative degrees. Then we may take $\operatorname{tm} f(\Gamma)$ to be the connective cover of $\operatorname{Tm} f(\Gamma)$ and obtain

$$
\operatorname{tmf}(\Gamma)_{*}=H^{0}\left(\overline{\mathfrak{M}}_{\text {ell }}(\Gamma) ; \omega^{\otimes * / 2}\right) .
$$

However if $H^{1}$ does not have the vanishing property, it may be much trickier to construct $\operatorname{tm} f(\Gamma)$ with this property. In specific examples, it could be done by hand, by killing the extra homotopy groups of the connective cover, as Hill-Lawson [HL10] and Lawson [Law15] do for the similarly behaved topological automorphic forms 
of discriminants 6 and 15. However we come by it, we assume the existence of a spectrum $\operatorname{tm} f(\Gamma)$ realizing the $H^{0}$ part, and a map $\operatorname{tm} f(\Gamma) \longrightarrow \operatorname{Tm} f(\Gamma)$ inducing a monomorphism on homotopy groups.

We continue taking $\mathfrak{n}$ to be the ideal of positive degree elements of $\mathbf{r}_{*}$, and in our cases this is a finitely generated ideal so that we can make the localization $\operatorname{tm} f(\Gamma) \longrightarrow \check{C}_{\mathfrak{n}} t m f(\Gamma)$.

Lemma 5.2. The map $\ell: \operatorname{tm} f(\Gamma) \longrightarrow T m f(\Gamma)$ induces an equivalence

$$
\check{C}_{\mathfrak{n}} t m f(\Gamma) \simeq \operatorname{Tmf}(\Gamma) .
$$

Proof: For brevity, let $\mathbf{t}=\operatorname{tm} f(\Gamma)$, and $\mathbf{T}=\operatorname{Tm} f(\Gamma)$; we show that $\ell: \mathbf{t} \longrightarrow \mathbf{T}$ has the universal property that $\mathbf{t} \rightarrow \check{C}_{\mathfrak{n}} \mathbf{t}$ enjoys.

First, note that if $x \in \mathfrak{n}$, then $\ell$ induces

$$
\mathbf{T}[1 / x] \simeq \mathbf{t}[1 / x]
$$

since the fibre of $\ell$ is bounded above.

Let $\mathbf{t} / \mathbf{x}$ be the unstable Koszul complex for some set $\mathbf{x}$ of radical generators of $\mathfrak{n}$. It remains to show that

$$
[\mathbf{t} / \mathbf{x}, \mathbf{T}]_{*}^{\mathbf{t}}=0
$$

For this we note that $\overline{\mathfrak{M}}=\overline{\mathfrak{M}}_{\text {ell }}(\Gamma)$ has a finite open cover by substacks $\overline{\mathfrak{M}}[1 / y]$ for $y \in \mathfrak{n}$, and the intersections of these are of the same form. (For example, we can pull back the cover of $\overline{\mathfrak{M}}_{\text {ell }}$ by the opens where the modular forms $c_{4}$ and $\Delta^{24}$ are respectively invertible.) Furthermore,

$$
\mathbf{T}[1 / y] \simeq \Gamma\left(\overline{\mathfrak{M}}[1 / y] ; \mathcal{O}^{\text {top }}\right)
$$

By our assumptions,

$$
H^{0}\left(\overline{\mathfrak{M}}[1 / y] ; \omega^{\otimes * / 2}\right)=\mathbf{t}_{*}[1 / y] .
$$

Since $y$ acts nilpotently on $\mathbf{t} / \mathbf{x}$, we see that

$$
[\mathbf{t} / \mathbf{x}, \mathbf{T}[1 / y]]_{*}^{\mathbf{t}}=0
$$

Since $\overline{\mathfrak{M}}$ has a cover whose sets and intersections are all of the form $\overline{\mathfrak{M}}[1 / y]$ it follows that $\mathbf{T}$ is built from the spectra $\mathbf{T}[1 / y]$, and hence

$$
[\mathbf{t} / \mathbf{x}, \mathbf{T}]_{*}^{\mathbf{t}}=0
$$

as required. 
5.D. Tabulation of examples. It is helpful to tabulate a range of examples we can deal with by these elementary means (i.e. where the coefficient ring is polynomial or a complete interesection).

The first entry in the row is the common name for the ring spectrum, either topological modular forms with a level $\Gamma$ structure $\operatorname{tm} f(\Gamma)$ (general reference [HL16]) or a particular ring of topological automorphic forms with additional data (general reference [BL10]). Each row is a $p$-local or $p$-complete statement, where $p$ is the second entry. The third column gives a finite group of automorphisms of $\mathbf{r}$. The homotopy fixed point spectrum will usually have much more complicated homotopy groups, which may be studied by descent. The degrees of generators are self-explanatory and $a$ is the Gorenstein shift.

\begin{tabular}{lcc|rrr|r||r|}
$\mathbf{r}$ & $p$ & & $\operatorname{deg}(x)$ & $\operatorname{deg}(y)$ & $\operatorname{deg}(z)$ & $\operatorname{deg}(f)$ & $a$ \\
\hline $\operatorname{tmf}(3)$ & 2 & $B T_{48}$ & 2 & 2 & - & - & -6 \\
$\operatorname{tm} f_{1}(3)$ & 2 & $C_{2}$ & 2 & 6 & - & - & -10 \\
$\operatorname{tm} f(2)$ & 3 & $\Sigma_{3}$ & 4 & 4 & - & - & -10 \\
$\operatorname{tm} f_{0}(2)$ & 3 & & 4 & 8 & - & - & -14 \\
\hline$t a f_{\delta 6}$ & 5 & two $C_{2}$ & 8 & 12 & 24 & 48 & 2 \\
$\operatorname{taf} f_{\delta 6}^{A L \alpha}$ & 5 & & 8 & 24 & 24 & 48 & -10 \\
$\operatorname{taf} f_{\delta 6}^{A L \beta}$ & 5 & & 8 & 12 & - & - & -22 \\
$\operatorname{taf} f_{\delta 6}$ & $\pm 1 \bmod 24$ & $C_{2} \times C_{2}$ & 8 & 12 & 24 & 48 & 2 \\
$\operatorname{ta} f_{\delta 6}^{A L \alpha}$ & $\pm 1 \bmod 24$ & & 16 & 24 & 44 & 88 & 2 \\
\hline$t a f_{\delta 14}$ & 3 & & 4 & 16 & - & - & -22 \\
\hline$t a f_{\delta 10, \sqrt{2}}$ & 3 & $C_{3}$ & 4 & 4 & 12 & 24 & 2 \\
\hline$t a f_{\delta 15}$ & 2 & $C_{8} \times C_{2}$ & 2 & 6 & 12 & 24 & 2 \\
\hline
\end{tabular}

Although the general features are covered above, we make four cases explicit. The details of the first set of examples (topological modular forms) can be found in a number of sources, including [MR09, Sto12, Sto14. The second set (topological automorphic forms of discriminant 6), which we summarize below, is based on the work of Hill-Lawson [HL10], as are the next two, topological automorphic forms of discriminants 14 and 10. The last charted example, topological automorphic forms of discriminant 15, is the subject of Lawson's paper [Law15].

Example 5.3. We consider the spectrum $\mathbf{r}=t a f_{\delta 6}$ which is the connective version of the spectrum $T a f_{\delta 6}=\check{C}_{\mathfrak{n}} \mathbf{r}$ of topological automorphic forms of discriminant 6 [HL10, Section 3]. Note that $a=2$ in this case, but as is done in [HL10, one can still construct a good connective spectrum $\mathbf{r}$ such that the analogue of Lemma 5.2 holds.

The coefficients are

$$
\mathbf{r}_{*}=\left(t a f_{\delta 6}\right)_{*}=K[x, y, z] /(f), \text { where } f=3 x^{6}+y^{4}+3 z^{2},
$$


with

$$
K=\mathbb{Z}[1 / 6],|x|=8,|y|=12,|z|=24 \text { and }|f|=48 .
$$

Thus $\mathbf{r}_{*}$ is a relative complete intersection, and $\mathbf{r}_{*}$ is Gorenstein. Indeed, it is easy to calculate local cohomology, directly or by local duality to see

$$
H_{\mathfrak{n}}^{*}\left(\mathbf{r}_{*}\right)=H_{\mathfrak{n}}^{2}\left(\mathbf{r}_{*}\right)=\Sigma^{a} \mathbf{r}_{*}^{\vee}
$$

where $\mathbf{r}_{*}^{\vee}=\operatorname{Hom}_{K}\left(\mathbf{r}_{*}, K\right)$ and $a=48-(8+12+24)-2=2$. Since this is in a single cohomological degree we have

$$
\pi_{*}\left(\Gamma_{\mathfrak{n}} \mathbf{r}\right)=\Sigma^{2} \mathbf{r}_{*}^{\vee},
$$

and then the cofibre sequence

$$
\Gamma_{\mathfrak{n}} \mathbf{r} \longrightarrow \mathbf{r} \longrightarrow \check{C}_{\mathfrak{n}} \mathbf{r}
$$

gives

$$
\pi_{*}\left(\check{C}_{\mathfrak{n}} \mathbf{r}\right)=\mathbf{r}_{*} \oplus \Sigma^{3} \mathbf{r}_{*}^{\vee},
$$

where the splitting follows by degree and parity.

Example 5.4. Considering the completion at $p=5$, there are two distinct lifts of the Atkin-Lehner involution $w_{6}$ on $t a f_{\delta 6}$, as in [HL10, Example 3.12]; for brevity, we call them $\alpha$ and $\beta$.

(1) The $\alpha$-involution negates $y$, so that $Y=y^{2}$ is invariant; $x$ and $z$ are fixed. We take

$$
\mathbf{r}:=\operatorname{ta} f_{\delta 6}^{A L \alpha}:=\left(\operatorname{ta} f_{\delta 6}\right)^{h C_{2}, \alpha} .
$$

Since 2 is invertible, the invariants give the homotopy

$$
\begin{gathered}
\mathbf{r}_{*}=\left(t a f_{\delta 6}^{A L \alpha}\right)_{*}=K[x, Y, z] /(f) \text { where } f=3 x^{6}+Y^{2}+3 z^{2}, \\
K=\mathbb{Z}_{5}^{\wedge},|x|=8,|Y|=24,|z|=24 \text { and }|f|=48 .
\end{gathered}
$$

This ring is Gorenstein of shift $a=48-(8+24+24)-2=-10$.

(2) The $\beta$-involution negates $z$, leaving $x$ and $y$ fixed. We take

$$
\mathbf{r}:=\operatorname{ta} f_{\delta 6}^{A L \beta}:=\left(\operatorname{ta} f_{\delta 6}\right)^{h C_{2}, \beta} .
$$

Again, since 2 is invertible, the invariants give the homotopy

$$
\mathbf{r}_{*}=\left(t a f_{\delta 6}^{A L \beta}\right)_{*}=K[x, y],
$$

where

$$
K=\mathbb{Z}_{5}^{\wedge},|x|=8 \text { and }|y|=12 .
$$

The ring $\mathbf{r}_{*}$, and hence also $\mathbf{r}$ is Gorenstein of shift $a=-(8+12)-2=-22$.

The difference in shifts in (1) and (2) illustrates that the change in shift on descent depends on the action. 
Example 5.5. Completing at a prime $p \equiv \pm 1 \bmod 24$, there are two commuting Atkin-Lehner involutions on $t a f_{\delta 6}$ and we may take $C_{2} \times C_{2}$ invariants, as in [HL10, Example 3.11]. We find that $X=x^{2}, Y=y^{2}$ and $T=x y z$ are invariant. We take

$$
\mathbf{r}:=\operatorname{ta} f_{\delta 6}^{A L \alpha \beta}:=\left(\operatorname{ta} f_{\delta 6}\right)^{h\left(C_{2} \times C_{2}\right)} .
$$

Again, since 2 is invertible, the invariants give the homotopy

$$
\begin{gathered}
\mathbf{r}_{*}=\left(t a f_{\delta 6}^{A L \alpha \beta}\right)_{*}=K[X, Y, T] /(g) \text { where } g=x^{2} y^{2} f=3 X^{4} Y+X Y^{3}+3 T^{2}, \\
K=\mathbb{Z}_{p}^{\wedge},|X|=16,|Y|=24,|T|=44 \text { and }|g|=88 .
\end{gathered}
$$

Thus $\mathbf{r}_{*}$ and hence also $\mathbf{r}$ itself is Gorenstein of shift $a=88-(16+24+44)-2=2$.

\section{INVARIANT THEORY AND DESCENT}

We imagine given a connective ring spectrum $\mathbf{r}$ and $\mathbf{R}=\check{C}_{\mathfrak{n}} \mathbf{r}$, and that a finite group $G$ acts on $\mathbf{r}$ and hence on $\mathbf{R}$. In fact we suppose that $\mathbf{r}$ and $\mathbf{R}$ are $G$-spectra, but we will only make use of the naive action. In the examples we know, $\mathbf{R}^{G} \longrightarrow \mathbf{R}$ is a Galois extension, so that in particular $\mathbf{R}^{G} \simeq \mathbf{R}^{h G}$, and $\mathbf{r}^{G}$ is the connective cover of $\mathbf{R}^{G}$.

We assume that it has been proved that the ring spectrum $\mathbf{r}$ has Gorenstein duality and $\mathbf{R}$ is Anderson self-dual, and we are interested in proving the good properties descend to $\mathbf{r}^{G}$ and $\mathbf{R}^{G}$. It is well known in algebra that the Gorenstein property need not descend to rings of invariants, and that when it does, there will usually be a change (the Solomon Supplement) in the Gorenstein shift.

Since rationalization commutes with taking invariants, one can learn about the general question of descent by considering the rational case, which is essentially algebraic. In particular, if $\mathbf{r}^{G}$ is Gorenstein with Solomon Supplement $b$ then this will also be true rationally, so we obtain a necessary condition for $\mathbf{r}^{G}$ to be Gorenstein and a prediction of its Gorenstein shift.

The purpose of this section is to describe what happens in the algebraic case, and to note that the Solomon Supplement is predicted from Solomon's Theorem in invariant theory, and can be calculated from the character of the action of $G$ on the polynomial generators.

Since the Čech and homotopy fixed point constructions commute with localization we assume for the remainder of this section that $\mathbf{r}_{*}$ is rational.

We thank D.J.Benson for his illustrated tutorials and we recommend [Ben93] for an account of the relevant invariant theory.

6.A. Context. In invariant theory, the best understood case is that of a polynomial ring, so let us assume $\mathbf{r}_{*}$ is a polynomial ring

$$
\mathbf{r}_{*}=K\left[x_{1}, \ldots, x_{r}\right],
$$


in $r>0$ variables, where $x_{i}$ is of even degree $d_{i}>0$ and $K$ is a $\mathbb{Q}$-algebra. Of course $\mathbf{r}_{*}$ (and hence $\mathbf{r}$ itself) is Gorenstein of shift

$$
a=-\left(d_{1}+\cdots+d_{r}\right)-r .
$$

Since the degrees $d_{i}$ are positive, $a \leq-2$, and we have (additively)

$$
\check{C}_{\mathfrak{n}}(\mathbf{r})_{*}=\mathbf{r}_{*} \oplus \Sigma^{a+1} \mathbf{r}_{*}^{\vee}
$$

giving an Anderson self-duality shift of $a+1$.

It is convenient to collect together the polynomial generators and say

$$
\mathbf{r}_{*}=K[V]
$$

where $V=Q\left(\mathbf{r}_{*}\right)$ is the indecomposable quotient, a graded free $K$-module.

6.B. Invariant theory. Now suppose $G$ acts on $\mathbf{r}$ in such a way that $\mathbf{r}_{*}$ is the symmetric algebra on a $K$-representation $V$ of $G$. If we now assume that $G$ acts by pseudoreflections, the Shephard-Todd theorem [Ben93, 7.2.1] states that $\mathbf{r}_{*}^{G}$ is also a polynomial ring

$$
\mathbf{r}_{*}^{G}=K\left[f_{1}, \ldots, f_{r}\right]
$$

where $f_{i}$ is of degree $e_{i}$. Watanabe's Theorem [Ben93, 4.6.2] follows easily from the local cohomology of polynomial rings

$$
H_{\mathfrak{n}}^{r}\left(\mathbf{r}_{*}\right)=\Sigma^{-\left(d_{1}+\cdots+d_{r}\right)} \mathbf{r}_{*}^{\vee} \otimes \operatorname{det}
$$

where det is the determinant of $V$. By Solomon's Theorem [Ben93, 7.3.1], we have

$$
\Sigma^{b}\left(\mathbf{r}_{*} \otimes \operatorname{det}\right)^{G}=\mathbf{r}_{*}^{G},
$$

where the Solomon Supplement is $b=\left(d_{1}+\cdots+d_{r}\right)-\left(e_{1}+\cdots+e_{r}\right)$. Hence we find

- $\mathbf{r}_{*}^{G}$ (and hence $\mathbf{r}^{G}$ ) is Gorenstein of shift $a+b$

- $\check{C}_{\mathfrak{n}}(\mathbf{r})_{*}^{G}$ (and hence $\check{C}_{\mathfrak{n}}(\mathbf{r})^{h G}$ ) has Anderson self-duality of shift $a+b+1$

Without any restriction on the action, the Hilbert series of the ring of invariants may be calculated by character theory from Molien's Theorem [Ben93, 2.5.2]:

$$
p\left(K[V]^{G}, t\right)=\frac{1}{|G|} \sum_{g \in G} \frac{1}{\operatorname{det}\left(1-g^{-1} t, V\right)} .
$$

This gives an alternative method for finding the degrees of generators if the invariants are polynomial. A more direct route to finding the Gorenstein shift is directly from the Hilbert series: Stanley's Theorem [Sta78, Theorem 5.5] shows that if $A$ is a Gorenstein graded ring of shift $a$, free over $K$ of Krull dimension $r$, the shift can be deduced from the functional equation

$$
p(A, 1 / t)=(-1)^{r} t^{r-a} p(t),
$$

where $r$ is the Krull dimension. 
6.C. Examples. We make explicit two well-known and rather simple examples.

Example 6.1. $\left(\mathbf{r}=k u, K=\mathbb{Z}, G=C_{2}\right)$. We have $\mathbf{r}_{*}=\mathbb{Z}[v]$ with $v$ of degree 2 . This is polynomial, and hence Gorenstein of shift $a=-3$, and it follows that $k u \longrightarrow H \mathbb{Z}$ is Gorenstein of shift -3 . In this case $\mathfrak{n}=(v)$ so that $\check{C}_{\mathfrak{n}} k u \simeq k u[1 / v]=K U$. We can then immediately deduce from Proposition 4.1 that $K U$ is Anderson self-dual of shift -2 .

Now consider connective real $K$-theory $k_{o}$ with the more complicated coefficient ring $k o_{*}=\mathbb{Z}[\eta, \alpha, \beta] /\left(2 \eta, \eta^{3}, \alpha^{2}=4 \beta\right)$ where $|\eta|=1,|\alpha|=4,|\beta|=8$. To show it is Gorenstein, we can use the fact that by Wood's theorem $\operatorname{Hom}_{k o}(k u, k o) \simeq \Sigma^{-2} k u$ and therefore $k_{o}$ is Gorenstein of shift $-3-2=-5$. Alternatively we can use the fact that $K O \longrightarrow K U$ is Galois and deduce that $K O=K U^{h C_{2}}$ is Gorenstein by descent. One can do this integrally by looking at the descent spectral sequence, but we will not give details here. (The interested reader could consult [HS14].) From this we infer that $K O$ is Anderson self-dual of shift -4 . The fact that ko has Gorenstein duality of shift -5 then follows by Lemma 4.3 .

For the present we will be satisfied to observe the rational result, which in particular tells us that the Solomon Supplement is -2 .

The action is that $C_{2}$ acts to negate $v$ so that $V$ is the sign representation $\tilde{\mathbb{Z}}$. We have that

$$
H_{\mathfrak{n}}^{*}\left(k u_{*}\right)=H_{\mathfrak{n}}^{1}\left(k u_{*}\right)=\mathbb{Z}\left[v, v^{-1}\right] / \mathbb{Z}[v]=\Sigma^{-2} v^{-1} \cdot \mathbb{Z}[v]^{\vee},
$$

and we see $\left(\mathbf{r}_{*} \otimes \operatorname{det}\right)^{G} \cong \Sigma^{2} \mathbf{r}_{*}^{G}$, so that $b=-2$.

Rationalisation gives $\mathbf{r}_{*}^{G}=\mathbb{Q}[v]^{G}=\mathbb{Q}\left[v^{2}\right]$. By inspection this is Gorenstein of shift -5 , and we see this is also $a+b$ as predicted above.

Example 6.2. $\left(\mathbf{r}=\operatorname{tm} f(2), K=\mathbb{Z}_{(3)}\right.$ and $\left.G=G L_{2}(3) \cong \Sigma_{3}\right)$. We have $\mathbf{r}_{*}=K[x, y]$ with $x$ and $y$ of degree 4 . This is polynomial, and hence Gorenstein of shift $a=-10$.

The action is that $V_{K}=\operatorname{ker}(K\{\underline{3}\} \longrightarrow K)$, where $K\{\underline{3}\}$ is the permutation representation associated to $\underline{3}=\{1,2,3\}$ with the standard action of $\Sigma_{3}$ (we will write [1], [2], [3] for the standard basis). Of course

$$
\begin{aligned}
H_{\mathfrak{n}}^{*}\left(\mathbf{r}_{*}\right) & =H_{\mathfrak{n}}^{2}(K[x, y])=K\left[x, x^{-1}, y, y^{-1}\right] /\left(K\left[x, x^{-1}, y\right]+K\left[x, y, y^{-1}\right]\right) \\
& =\Sigma^{-8}(K[x, y] \otimes \operatorname{det})^{\vee} .
\end{aligned}
$$

We now rationalize to apply the above theory. There are three simple rational representations, $\epsilon$, det and $V$, of dimensions 1,1 , and 2 , where $V=\operatorname{ker}(\mathbb{Q}\{\underline{3}\} \longrightarrow$ $\mathbb{Q}) \cong V_{K} \otimes \mathbb{Q}$. It is routine to calculate the decomposition of the symmetric powers into these simple representations of $\Sigma_{3}$. Writing $(i j k)$ for $i \epsilon \oplus j \operatorname{det} \oplus k V$, the decompositions of the first six symmetric powers of $V_{K}$ (in degrees $0,4,8,12,16$, 20) are (100), (001), (101), (111), (102), (112). The rest follow by the fact that if the part in degree $4 d$ decomposes as $(i j k)$ the part in degree $4 d+24$ decomposes as $((i+1)(j+1)(k+1))$. 
The dimension of the invariants is just the number of copies of $\epsilon$ which is thus

$$
101111212222323333 \ldots
$$

If we take $x=[1]-[2]$ and $y=[2]-[3]$ it is easy to find the invariants $A=$ $x^{2}+x y+y^{2}=N(-x y)$ of degree 8 and $B=x^{3}-y^{3}-3 x y(x+y) / 2$ of degree 12, giving $\mathbf{r}_{*}^{\Sigma_{3}}=K[A, B]$. This (and hence $\mathbf{r}^{h G}$ rationally) is Gorenstein of shift -22 , and $\mathscr{C}_{\mathfrak{n}}(\mathbf{r})^{h G}$ is rationally Anderson self-dual of shift -21 .

On the other hand Solomon's theorem shows that $\left(\mathbf{r}_{*} \otimes \operatorname{det}\right)^{\Sigma_{3}}=\Sigma^{12} \mathbf{r}_{*}^{\Sigma_{3}}$. We note that $-b=12=(12-4)+(8-4)$ as expected.

\section{REFERENCES}

[And69] D.W. Anderson, Universal coefficient theorems for $k$-theory, mimeographed notes, Univ. California, Berkeley, Calif. (1969).

[BC76] Edgar H. Brown, Jr. and Michael Comenetz, Pontrjagin duality for generalized homology and cohomology theories, Amer. J. Math. 98 (1976), no. 1, 1-27. MR 0405403

[Ben93] D. J. Benson, Polynomial invariants of finite groups, London Mathematical Society Lecture Note Series, vol. 190, Cambridge University Press, Cambridge, 1993. MR 1249931

[BL10] Mark Behrens and Tyler Lawson, Topological automorphic forms, Mem. Amer. Math. Soc. 204 (2010), no. 958, xxiv+141. MR 2640996

[DGI06] W. G. Dwyer, J. P. C. Greenlees, and S. Iyengar, Duality in algebra and topology, Adv. Math. 200 (2006), no. 2, 357-402. MR 2200850

[DGI11] W. G. Dwyer, J. P. C. Greenlees, and S. B. Iyengar, Gross-Hopkins duality and the Gorenstein condition, J. K-Theory 8 (2011), no. 1, 107-133. MR 2826281

[EKMM97] A. D. Elmendorf, I. Kriz, M. A. Mandell, and J. P. May, Rings, modules, and algebras in stable homotopy theory, Mathematical Surveys and Monographs, vol. 47, American Mathematical Society, Providence, RI, 1997, With an appendix by M. Cole. MR 1417719

[GM95] J. P. C. Greenlees and J. P. May, Completions in algebra and topology, Handbook of algebraic topology, North-Holland, Amsterdam, 1995, pp. 255-276. MR 1361892

[GM16] J. P. C. Greenlees and Lennart Meier, Gorenstein duality for Real spectra, arXiv:1607.02332 (2016).

[Gre93] J. P. C. Greenlees, K-homology of universal spaces and local cohomology of the representation ring, Topology 32 (1993), no. 2, 295-308. MR 1217070

[HL10] Michael Hill and Tyler Lawson, Automorphic forms and cohomology theories on Shimura curves of small discriminant, Adv. Math. 225 (2010), no. 2, 1013-1045. MR 2671186

[HL16] _ Topological modular forms with level structure, Invent. Math. 203 (2016), no. 2, 359-416. MR 3455154

[HM17] Michael Hill and Lennart Meier, The C2-spectrum Tmf1(3) and its invertible modules, Algebr. Geom. Topol. 17 (2017), no. 4, 1953-2011. MR 3685599

[HS14] Drew Heard and Vesna Stojanoska, K-theory, reality, and duality, J. K-Theory 14 (2014), no. 3, 526-555. MR 3349325

[Law15] Tyler Lawson, The Shimura curve of discriminant 15 and topological automorphic forms, Forum Math. Sigma 3 (2015), e3, 32. MR 3324940 
[Mei16] Lennart Meier, (topological) modular forms with level structures: decompositions and duality, arXiv:1609.09264 (2016).

[MR99] Mark Mahowald and Charles Rezk, Brown-comenetz duality and the adams spectral sequence, Amer. J. Math 121 (1999), no. 6, 1153-1177.

[MR09] _ Topological modular forms of level 3, Pure Appl. Math. Q. 5 (2009), no. 2, Special Issue: In honor of Friedrich Hirzebruch. Part 1, 853-872. MR 2508904

[Sta78] Richard P. Stanley, Hilbert functions of graded algebras, Advances in Math. 28 (1978), no. 1, 57-83. MR 0485835

[Sto11] Vesna Stojanoska, Duality for Topological Modular Forms, ProQuest LLC, Ann Arbor, MI, 2011, Thesis (Ph.D.)-Northwestern University. MR 2890200

[Sto12] ㄴ Duality for topological modular forms, Doc. Math. 17 (2012), 271-311. MR 2946825

[Sto14] _ C Calculating descent for 2-primary topological modular forms, An alpine expedition through algebraic topology, Contemp. Math., vol. 617, Amer. Math. Soc., Providence, RI, 2014, pp. 241-258. MR 3243402

School of Mathematics and Statistics, Hicks Building, Sheffield S3 7RH. UK.

E-mail address: j.greenlees@sheffield.ac.uk

Department of Mathematics, University of Illinois, 1409 W Green St., Urbana IL 61802, USA.

E-mail address: vesna@illinois.edu 